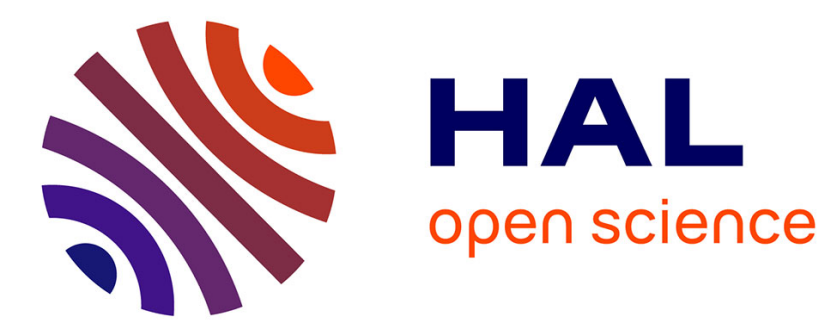

\title{
Recherches en télédétection spatiale au CIMA
}

Isabelle Anglade, Gilles Selleron, Jacques Hubschman

\section{To cite this version:}

Isabelle Anglade, Gilles Selleron, Jacques Hubschman. Recherches en télédétection spatiale au CIMA. Revue Géographique des Pyrénées et du Sud-Ouest, 1986, 57 (3), pp.472-480. 10.3406/rgpso.1986.4964 . hal-02780703

\section{HAL Id: hal-02780703 \\ https://hal-univ-tlse2.archives-ouvertes.fr/hal-02780703}

Submitted on 4 Jun 2020

HAL is a multi-disciplinary open access archive for the deposit and dissemination of scientific research documents, whether they are published or not. The documents may come from teaching and research institutions in France or abroad, or from public or private research centers.
L'archive ouverte pluridisciplinaire HAL, est destinée au dépôt et à la diffusion de documents scientifiques de niveau recherche, publiés ou non, émanant des établissements d'enseignement et de recherche français ou étrangers, des laboratoires publics ou privés. 


\section{Recherches en télédétection spatiale au CIMA}

Isabelle Anglade, Gilles Selleron, Jacques Hubschman

Citer ce document / Cite this document :

Anglade Isabelle, Selleron Gilles, Hubschman Jacques. Recherches en télédétection spatiale au CIMA. In: Revue géographique des Pyrénées et du Sud-Ouest, tome 57, fascicule 3, 1986. L'élément et le système. pp. 472-480; doi : https://doi.org/10.3406/rgpso.1986.4964

https://www.persee.fr/doc/rgpso_0035-3221_1986_num_57_3_4964

Fichier pdf généré le 06/04/2018 
filière bois dans le Tarn où les originaires de Mazamet jouent un rôle primordial; mais ses projets ne se sont pas encore concrétisés. D'autres études sont conduites dans l'Association pour le développement économique et social des Mont de Lacaune mais se heurtent souvent à l'immobilisme des entrepreneurs locaux qui n'osent se lancer vraiment dans des actions d'envergure.

En fait, l'attentisme semble encore l'emporter à l'aval de la "filièrebois tarnaise ", si tant est qu'elle existe. Voilà qui n'est pas fait pour diminuer le relatif pessimisme constaté chez certains reboiseurs qui estiment que leur production une fois parvenue à maturité ne trouvera preneur qu'au prix du marché international. Il est loin d'être sûr qu'ils puissen alors en vivre. Les habitants des hautes terres tarnaises, après avoir perdu la maîtrise de leur espace de vie, risquent fort de ne pouvoir non plus contrôler les débouchés espérés pour les résineux qui ont massivement colonisé la Montagne Noire et les Monts de Lacaune. En ce sens la politique conduite par le Fond forestier national dans le Tarn ne semble pas avoir réussi.

Frédéric OGé.

\section{RECHERCHES EN TÉLÉDETECTION SPATIALE AU CIMA}

Le CIMA est tard venu à la télédétection. A partir de 1982 cependant, la proximité géographique du CNES et du GDTA a facilité les collaborations et permis le démarrage d'un programme d'étude des données NOAA. En 1984, d'autre part, est attaché au CIMA un chercheur expérimenté, si bien que désormais, les recherches en télédétection sont largement engagées: trois domaines sont en cours de prospection: agriculture, forêt montagnarde, steppe arbustive tropicale.

\section{Applications agricoles de l'interprétation des images NOAA.}

L'étude du couvert végétal et, plus particulièrement, de l'espace agricole à partir des images des satellites de la série NOAA, reste peu usitée. En effet, une faible résolution spatiale $\left(1 \mathrm{~km}^{2}\right)$ interdit généralement l'observation de parcelles agricoles dont la taille movenne, en Europe, est souvent de l'ordre de l'hectare.

Le capteur des satellites NOAA a, en revanche, une propriété fort intéressante pour l'étude du couvert végétal : la haute répétitivité des enregistrements, qui permet d'obtenir deux images par jour. Il en résulte que la probabilité de disposer d'images claires (sans nuages) à intervalles do temps très court (quelques jours), est beaucoup plus élevée que pour les satellites à haute résolution spatiale, ce qui en fait un instrument bien adapté au suivi des cultures. A cela s'ajoute la possibilité d'analyse thermographique des terroirs cultivés, grâce au canal infrarouge thermique des NOAA. 
Compte tenu de ces caractéristiques et des possibilités de surveillance continue des cultures au cours des campagnes agricoles, un programme de recherche spécifique a été mis en place et conduit en collaboration avec le Laboratoire d'études et de recherches en télédétection spatiale; il associe divers organismes de la profession agricole (Chambre d'agriculture de l'Aude, Institut technique des céréales et fourrages, Association générale des producteurs de maïs, AZF/Charbonnages de France, Centre technique interprofessionnel des oléagineux métropolitains). Ce programme porte, dans l'immédiat, sur une région s'étendant du Béarn et de la Gascogne, jusqu'au littoral méditerranéen. Les recherches s'organisent autour de trois axes complémentaires : identification, suivi et cartographie des principales espèces cultivées; étude de l'interaction des paramètres de surface et des paramètres métćorologiques dans le sigral thermique; analyse et cartographie du comportement thermique des terroirs agricoles.

a. Identification, suivi et cartographie des cultures. - La méthode repose, fondamentalement, sur la mesure séquentielle de la réponse radiométrique des cultures, à travers le calcul de l'indice de végétation. Cet indice varie, pour l'essentiel, avec le développement de la biomasse verte des cultures. Compte tenu de la résolution spatiale de NOAA (100 ha), la valeur de lindice de végétation d'un pixel donné représente la moyenne des indices de végétation de toutes les cultures coexistant, à un moment donné, sur une surface de 100 ha. Le problème est donc d'identifier, dans cette valeur moyenne, la réponse propre à chaque culture. Pour cela, il faut s'appuyes. sur un échantillonnage de référence et sur un modèle d'évolution de la réponse des cultures.

Les aires d'échantillonnage, choisies dans l'ensemble de la région étudiéc de façon à représenter au mieux la variété des conditions climatiques, édaphiques et d'occupation agricole du sol, doivent en outre, répondre à des critères de taille et d'occupation agricole adaptés à la résolution spatiale des NOAA. Leur superficie est en général de 1000 à 2000 hectares, taille compatible avec la qualité des corrections géométriques. Ces aires ont également été sélectionnées afin de correspondre, dans la mesure du possible, à une gamme progressive d'occupation du sol par chaque culture, les aires appartenant ainsi à une classe déterminée d'association culturale. Le suivi de ces aires d'échantillonnage permet d'extraire les signatures temporelles des diverses classes d'associations culturales en dressant les courbes d'évolution de leurs index de végétation. Pour aller plus loin et tenter d'identifier les signatures propres à chaque culture, il faut s'appuyer sur des modèles d'évolution de la réponse des cultures.

Les modèles d'évolution de la réponse des cultures s'appliquent à trois groupes d'espèces cultivées : céréales d'hiver (blé, orge), tournesol et maïs sorgho. Les cycles biologiques de ces groupes sont nettement décalés dans le temps, condition impérative pour l'identification. Pour chaque culture, on peut établir la courbe d'évolution du taux de couverture du sol par la biomasse verte, pondérée par la prise en compte de l'évolution de l'indice foliaire et par celle de la teneur en eau des tissus. La courbe temporelle d'indice de végétation de chaque culture (ou groupe des cultu. 
res) traduit, théoriquement, l'évolution ainsi établie du taux de couverture en biomasse verte.

Les premiers résultats ont été tirés de l'analyse de 17 scènes entre mars et octobre 1983; ils ont déjà permis de discriminer les cultures principales, à partir des images correspondant au maximum de réponses de chacune d'elles. La première application pratique de ces résultats est cartographique : localisation et répartition des principales cultures, (céréales d'hiver, tournesol, maïs/sorgho). L'intérêt majeur de ce produit est de pouvoir ètre fourni précocement, en cours (ou en fin) de campagne agricole et d être annuellement actualisable.

Les recherches se poursuivent dans deux directions: affiner les modèles d'évolution de la réponse des cultures, de façon à explorer la possibilité d'une meilleure discrimination des espèces cultivées et prendre en compte des cultures encore secondaires, mais dont l'importance économique est réelle (soja, colza, etc.); analyser en détail les nombreuses images des campagnes agricoles 1984 (25 images) et 1985 (47 images), avec des pas cle temps suffisamment faibles, pour interprêter l'évolution continue des réponses, une fois calculée l'occupation au sol de chaque culture. On pourra ainsi mieux définir les réponses radiométriques correspondant à des comportements culturaux particuliers, liés aux conditions bioclimatiques (sécheresse de 1985, par exemple) ou phytopathologiques de la campagne agricole.

L'objectif est de constituer un catalogue de réponses radiométriques qui, avec un recul de quelques années, couvre la plus large gamme de situations possibles et auquel on pourra se référer constamment. A plus long terme, le programme a l'ambition de mettre en place un système qui permette de suivre, en temps "presque" réel ( 5 à 10 jours après l'acquisition) les étapes de développement des cultures et de fournir des informations sur l'état, à un moment et à un endroit donnés, de la végétation cultivée.

b. Interaction des paramètres de surface et des paramètres météorologiques. - L'interprétation d'une image thermique implique nécessairement que la signification du signal thermique soit connue. En théorie, le signal thermique reflète la température de la surface (sol et végétation, dans notre cas). En fait, nos recherches précédentes ont montré que le signal thermique de NOAA était plus ou moins sensible aux conditions météorologiques. La méthode repose dans ses grandes lignes sur l'analyse du rapport entre la réponse thermique des aires d'échantillonnage (température radiométrique) et leur indice de végétation, d'une part, la température météorologique au sol, d'autre part. Une forte corrélation entre indice de végétation et température radiométrique traduit l'influence prépondérante des paramètres de surface, sol et végétation surtout. Une forte corrélation entre température radiométrique et température météorologique signale, au contraire, que la température radiométrique reflète, pour une bonne part, la température de l'air, elle-même liée à l'humidité atmosphérique.

Les résultats déjà acquis montrent que l'on peut mettrc en évidence une catégorie d'images caractérisées par une faible corrélation tempéra- 
ture radiométrique - indice de végétation, plus fréquentes en domaine méditerranéen qu'en domaine aquitain. En fait, la distinction entre images exprimant surtout la température de surface et images reflétant principalement l'influence de l'air, reste fragile. Les recherches en cours ont pour objectif de consolider et d'approfondir les premières données établies à partir d'analyses de corrélations plus nombreuses et plus fiables sur les images de 1984 et 1985.

c. Analyse et cartographie du comportement thermique des terroirs.

Les deux catégories d'images déjà évoquées n'offrent pas, quoique complémentaires, les mêmes possibilités.

Sur les images thermographiques traduisant surtout la réponse thermique de la surface, la cartographic du comportement thermique des terroirs peut être établie; elle révèle, le plus souvent, une mosaïque complexe qui met en évidence l'importance de paramètres tels que le degré de développement de la biomasse, la proportion de sol nu, les réserves hydriques du sol, l'irrigation... Ces zonages thermiques, effectućs à plusieurs dates successives, permettent des diagnostics localisés des conditions de terroir et rendent possible l'identification d'accidents culturaux ou de "stress" hydriques; parallèlement, ils peuvent apporter une aide spécifique à certains problèmes d'aménagement agricole (choix d'encépagement, par exemple) ou contribuer au perfectionnement de certaines méthodes agronomiques (aide à la méthode prévisionnelle de fertilisation azotće du blé).

Les images thermographiques exprimant la température de l'air donnent une bonne représentation "climatique " dont l'intérêt réside surtout dans la comparaison entre les principaux types de flux. L'influence de la végétation et de la surface du sol étant ici très réduite, on visualise plus clairement les effets climatiques locaux, qui intègrent en particulier les paramètres de relief et d'exposition et les effets d'abri.

Dans les deux cas, on s'attache aujourd'hui à constituer une base de données regroupant les réponses thermiques par type de temps, de façon à étudier les variations de ces réponses pour un même type de temps et à analyser leurs relations avec des paramètres météorologiques secondaires (vent, antécédent météorologique, etc.).

\section{Télédétection des forêts de montagne.}

L'étude par télédétection de la forêt montagnarde avec un champ d'appli. cation limité, pour l'instant, aux Pyrénées s'inscrit dans une problématique spécifique propre à la fois à la forêt et à la montagne.

D'une part, le contexte national peut se résumer ainsi : par des inventaires traditionnels, l'état de la forêt française est connu tous les 10 ans. Or, les satellites d'observation de la terre permettent un suivi régulier et pluriannuel des espaces forestiers. Mais les données qu'ils fournissent sont mal maîtrisées et pas encore exploitées de manière opérationnelle, alors que leurs performances s'améliorent (résolutions au sol, et bandes spectrales). 
D'autre part, les expérimentations et les développements méthodologiques menés depuis 10 ans en télédétection forestière ne concernaient à juste titre, dans un premier temps - que les régions de relief modéré et excluaient par conséquent environ le tiers du couvert forestier français situé en montagne.

Or l'évolution actuelle du milieu montagnard, est, on le sait, tout à fait spécifique : très affecté par l'exode rural, il connaît une mutation rapide principalement marquée dans le paysage par une déprise agricole et pastorale et un accroissement des friches et de la forêt. Le programme de recherche a donc un double objectif méthodologique et thématique: élaborer une nouvelle méthode d'interprétation en télédétection appropriéc aux zones de relief, tester l'apport de la nouvelle génération de satellites (Landsat - Thematic Mapper et Spot) et produire des inventaires actualisés - cartographiques et statistiques - sur l'état des massifs forestiers montagnards (essences, structures, régimes, aspect phytosanitaire, etc.) et sur leurs évolutions (coupes, incendies, " enfrichement", défrichement, etc.), tout en intégrant leurs marges composites (boisements lâches, landes, pelouses, prairies de fauche, etc.).

Ainsi, à moyen terme, la télédétection constituerait-elle un outil puissant pour observer les transformations du paysage montagnard et permettre aux aménageurs, forestiers, économistes, législateurs et autres décideurs de se saisir de cet instrument pour agir sur le terrain en temps opportun. a. Sites - tests et données satellitaires. - Avant d'inventorier l'ensemble du massif, deux sites tests des Pyrénées centrales et orientales ont été sélectionnés pour leurs représentativités forestières. L'un, sur le Pays de Sault près de Quillan, dans l'Aude, l'autre sur le massif du Couserans (Vallée du Garbet), en Ariège.

Sur chacun d'eux des simulations du futur satellite français Spot ont été enregistrées par avion respectivement en $1982-83$ et en 1983. Ces simulations, de bonnes qualités radiométriques, n'ont cependant pas étè corrigées géométriquement : c'est un handicap sérieux, surtout en montagne. Elles servent donc seulement de banc d'essai en vue de l'analyse plus fine des images de Landsat 5 - Thematic Mapper - (T.M.) - 26.6.1984 et 28.7.1984 acquises à ce jour - puis celles de Spot à partir de l'été 1986. b. La prise en compte des contraintes. - On peut relever deux types de contraintes. Le premier est inscrit dans le paysage lui-même, au relief très accidenté, par la très grande variation des conditions d'exposition des versants et par l'importance des pentes, qui peuvent atteindre 36" pour les forêts et dépasser $45^{\circ}$ pour les formations herbacées de l'étage subalpin.

Par ailleurs, l'hétérogénéité des forêts est bien marquée tant par les mélanges d'espèces, par la variation des traitements et des régimes, par la forme même des arbres tributaires de la pente, de l'exposition, du sol et de l'impact de l'homme que par le petit parcellaire morcelé dominant. L'étagement de la végétation, différencié d'une vallée à l'autre, apporte ses nuances à ce schéma contraignant.

Le deuxième type de contraintes est propre à la télédćtcction. Tout d'abord les satellites Landsat survolent les Pyrénées $\left(43^{\circ} \mathrm{N}\right)$ à $10 \mathrm{~h}$ TU. 
A cette heure, le soleil est encore bas sur l'horizon ce qui accroît d'autant les plages d'ombre sur les versants nord et ouest surtout, et par conséquent, ne permet pas de voir le terrain. Les confusions radiométriques qui en résultent sur les images, par exemple, entre l'ombre et l'cau (forte absorption du rayonnement dans l'infrarouge), sont encore accentuées si des sapinières sont installées sur ces mêmes versants, car les résineux absorbent également une grande part du rayonnement à la différence des feuillus. Spot passera vers les $11 \mathrm{~h}$ TU: les plages d'ombre seront moins ctendues en visée verticale ct peut-être l'adjonction de deux visées obliques Est et Ouest sur le même lieu pallieront en grande partie cet inconvénient majeur.

En plus de l'inadéquation des images en milieu de montagne, les méthodes ususlles en télédétection doivent s'améliorer pour se conformer aux progrès technologiques des satellites qui présentent maintenant des résolutions spatiales plus fines $(30 \mathrm{~m}$ pour Thematic Mapper; 20 et $10 \mathrm{~m}$ pour Spot) et plus de bandes spectrales (Thematic Mapper: 7, Spot: 4) étalées du visible au moyen infrarouge.

Les avantages qui en résultent par un rapprochement visuel entre image et photographie aćrienne créent également certains inconvénients encore non maîtrisés : le traitement statistique d'une scène Landsat doit désormais s'effectuer sur 39,5 millions de points par canal alors aue les logiciels étaient adaptés aux anciens Landsat 1,2 et 3 soit 7 millions de points par canal. De la même manière et en règle générale, si le passage du $80 \mathrm{~m}$ de résolution à 20 et $30 \mathrm{~m}$ améliors la perception de la structure des peuplements forestiers, il transforme aussi leurs textures de plus ou moins lisses à granuleuses et en conséquence accroît l'hétérogénéité de leurs réponses spectrales que révèle l'augmentation de la variance radiométrique d'un même thème.

Ces contraintes inhérentes à la télédétection, doublées de celles de la topographie font apparaitre des confusions radiométriques plus fortes entre les différents types d'occupation du sol. Résoudre l'ensemble de ces problèmes, impose d'orienter les recherches dans plusieurs directions pour mieux comprendre le comportement radiométrique des formations végétales in situ et mieux isoler les paramètres physiques (rayonnement, atmosphère, pente, exposition...) qui influent sur la mesure radiométrique. Une collaboration étroite entre thématiciens, physiciens et informaticiens a donc commencé en 1984; elle réunit l'IFN, le CIMA, le LERTS (CNES), le LSI et le CEMAGREF.

Ces organismes, moins le LSI, participent au projei PE.PS (Programme d'évaluation préliminaire de Spot) sur la télédétection forestière en montagne avec l'imagerie Spot. Les résultats de ces recherches sont attendus non seulement par les services forestiers (ONF, IFN) mais aussi par les Conseils régionaux de Midi-Pyrénées et Languedoc-Roussillon qui pourront être amenés à les soutenir.

c. Méthodologie. - L'évaluation des résultats médiocres d'un premier essai de classification supervisée de la simulation du Couserans (confusion entre forêt et landes, landes et friches et entre les essences forestières...) nous conduit à proposer une nouvelle méthodologic. 
Afin d'appréhender les confusions radiométriques, on a, tout d'abord, mis l'accent sur la méthode des relevés de terrain sur les sites des simulations. Une fiche analytique orientée vers la description physionomique de la végétation a été élaborée. Elle rend compte de manière humogène et codifiée du contenu (forêt, lande, prairie, pelouse, etc.), de la composition (espèces, etc.), des structures verticale et horizontale de chaque parcelle échantillon en y intégrant les conditions physiques de relief, pente et orientations des versants. La stratégie d'échantillonnage des parcelles (128 dans la vallée du Garbet) a été définie à la fois sur photographies aériennes IRC et sur le terrain afin d'obtenir le "maximum " de conditions physiques différentes par formation végétale.

Traitements et interprétations des images s'imbriquent autour de deux pôles de recherches complémentaires. Il s'agit, avant d'spérer n'importe quelle classification, d'une part, de comprendre le comportement radiométrique des formations végétales en montagne, et, d'autre part, de saisir l'influence de l'exposition des versants sur la réponse radiométrique, tant ce paramètre, extrêmement contraignant en télédétection, est en revanche immédiatement numérisable.

Une typologie des ressemblances et des confusions radiométriques sera établie d'après les analyses des signatures et diagrammes spectraux de l'ensemble des parcelles repérées. Elle permettra également de préciser l'apport des différentes longueurs d'onde et résolutions au sol des images Landsat-TM et Spot (dont les simulations). Ces résultats seront mis en correspondance avec les données exogènes du relief, d'où une première évaluation de l'effet du relief sur la radiométrie.

Parallèlement, une étude ponctuelle et plus physique sera expérimentée sur deux types de couvert forestier (feuillus et résineux) échantillonnés en fonction de l'incidence d'éclairement, de l'incidence d'observation et de l'azimut relatif entre ces deux directions. Les corrélations entre la variable topographique et la réponse physique (d'abord sur IRC puis sur image) seront dégagées et modélisées par des méthodes statistiques. En complément et compte tenu de ce que la topographie joue un rôle important dans la répartition des essences forestières et des formations végétales, la fréquence de cette distribution spatiale en fonction de l'altitude et de l'ensoleillement potentiel, sera étudiée avec les points d'inventaire de l'IFN. Ensuite, un "modèle numérique de terrain », en cours de réalisation au LERTS - LSI, sera appliqué sur les images TM (puis Spot) pour s'affranchir des conditions de relief. On procédera alors à l'estimation de sa qualité (recherche du meilleur intervalle de pas nécessaire en milieu accidenté) et de sa validité à l'aide des parcelles échantillons précé. clemment choisies et au moyen de matrices de confusion issues d'images classifiées. Avec le résultat de l'étude des fréquences de la répartition des formations végétales, ce "MNT » sera utilisé comme support de découpage du paysage en sites sensiblement homogènes. D'où un autre type d'évaluation. Puis, de nouvelles analyses des signatures et diagrammes spectraux sur les images corrigées par le "MNT» seront faites puis interprétées sur les images TM et Spot en visées verticale et oblique. 
Ainsi, par une meilleure appréhension des relations physiques et thématiques, des "cartes de végétation " à diverses échelles seront réalisées et actualisé:s par la mise en valeur des changements dàns les couverts forestiers et dans la distribution spatiale des formations végétales.

\section{La caatinga du Nordeste du Brésil et les données MSS Landsat.}

Sa bordure littorale exceptée, le Nordeste du Brésil appartient presque cn entier au domaine semi-aride tropical et sa mosaïque complexe de faciès végétaux relève d'un même ensemble phytugéographiçue : la steppe arbuslive (caatinga).

Comme dans tous les milieux semi-arides, la fragilité de la couverturc végéta!e et du sol, la sensibilité à la pression anthropique (défrichements, agriculture, pâturage), le retour périodique de trains d'annér's sèches, ont conduit à multiplier les observations et les diagnostic:; sur l'évolution des milieux et à proposer une surveillance continue à l'aide des movens satellitaires.

Cependant, l'analyse d'images-satellites de la couverture végétale s`miaride tropicale pose des problèmes spécifiques : fréquence de la nébulosité sur les scènıs, faiblesse de la biomasse verte par rapport à la biomassc totale, participation élevée de la réponse du sol... Il y a là un champ de recherches encore relativenent peu exploré. dont l'approfondissement est fondam'ntal pour l'avenir de l'utilisation de l'imagerie satellitaire dans ces domaines bioclimatiques.

Dans cette perspective qu'une recherche a été engagcée en collaboration avec l'Instituto nacional de pesquisas espaciais brésiliens. L'objectif de cette recherche est triple : - tester l'apport et les limites des données satellitaires (Landsat 1, 2 et 3 ) dans ces types de milieux; - élaborer un système de classification de la caatinga adapté à l'analvse des images; - évaluer. en comparant les données-images, les photos aériennes de trois missions successives $(1955,1965,1983)$ et les observations au sol, l'évolution de la caatinga, en particulier depuis 1973, première année pour laquelle on dispose de données satellitaires.

En accord avec les organismes de développement régional (SUDENE) ou de développement agricole (EMBRAPA. CPATSA), un site test a été choisi, à Quixabá, dans la partie occidentale de l'Etat de Pernambouc, comme assez représentatif du milieu semi-aride tropical nordestin et réunissant un minimum de conditions (accessibilité, topographie, données thématiques de base, etc.). Le programme de recherche tient en trois étapes: mise au point d'une classification structurale de la caatinga, établie à la fois par levés au sol et interprétation des données aériennes IRC de 1983; cartographie structurale multitemporelle de la caatinga, à partir des missions aériennes, et analyse des principaux traits de l'évolution au cours de la période 1954-1983; traitement des données Landsat-MSS de 1973 à 1983 en distinguant saisons sèches et saisons humides, années sèches et années humides. Le point central concerne les problèms de confusion radiométrique et les difficultés de discrimination entre classes thématiques. Sur ces bases, l'interprétation de l'évolution 1973 - 1983, propo- 
sée et discutée, est indissociable de l'évaluation des possibilités et limites de l'imagerie Landsat - MSS.

Cette recherche, qui devrait se terminer en 1986, se prolongerait par l'utilisation de modes de traitement encore non expérimentés dans ce milieu (texture) et par l'étude de données satellitaires nouvelles: TM et Spot, sur le même site-test ou sur des sites comparables, choisis en liaison avec l'NPE et les organismes de développement régional.

Les trois directions de recherche évoquées ci-dessus correspondent à des programmes engagés depuis un certain temps et à propos desquels on jouit déjà d'un certain recul. D'autres voies sont actuellement explo. rées par le biais de collaboration à certains projets. Ainsi, pour la conception d'une base de données "géocodées", aux fins d'aménagement régional: l'imagerie satellitaire y tient une place importante. De même la Conseil régional de Midi-Pyrénées vient-il de retenir une proposition d'étude du tracé routier de la future liaison Toulouse-Barcelone à partir d'images TM et/ou SPOT, à l'ana!yse desquelles collaborera le CIMA. Enfin, des projets à plus long terme se dessinent, où la télédétection est présente: Vénézuela, Brésil... Les éléments sont donc en place; scul manque encore le (micro) système de traitement d'images.

Isabelle Anglade, Gilles Selleron et Jacques Hubschman. 